\title{
Las revisiones sistemáticas como proceso de selección artificial del conocimiento científico en el área de salud $^{1}$
}

\author{
Systematic Reviews as process of artificial selection of scientific \\ knowledge in the healthcare area
}

Oscar Eliezer Mendoza De Los Santos ${ }^{2}$

Fecha de recepción: 19 de marzo de 2020

Fecha de aceptación: 11 de mayo de 2020

\begin{abstract}
1- Agradecimientos:
Al Consejo Nacional de Ciencia y Tecnología (CONACYT) por la beca otorgada para la realización de mi posgrado $\left(\mathrm{N}^{\circ}\right.$ de apoyo 750147). Al Dr. Adalberto De Hoyos Bermea, por sus observaciones y comentarios durante el desarrollo de este artículo.

2- Nacionalidad: Mexicana.Grado: Licenciado en Psicología por la Universidad Autónoma de Tamaulipas. Adscripción: Estudiante de la Maestría en Metodología de la Ciencia. CIECAS-Instituto Politécnico Nacional. (D ORCID: https://orcid. org/0000-0001-9448-4947. Correo electrónico: mendoza.oscar1990@gmail.com
\end{abstract}




\section{Resumen}

El presente ensayo tiene por objetivo conceptualizar y analizar, desde el marco de la epistemología evolucionista, a las revisiones sistemáticas como proceso de selección artificial del conocimiento científico en el área de salud. Primeramente, se analizan los supuestos de la evolución del conocimiento científico por selección natural. Posteriormente, se propone que las revisiones sistemáticas sean consideradas como una forma de metainvestigación, siendo esta última un tipo de investigación cuyo objeto de estudio es el conocimiento científico y la evaluación del mismo. Consecutivamente, se propone el concepto de Selección Artificial Epistémica, para referir a un proceso de selección epistémica distinto al del modelo de selección natural. Dado que las revisiones sistemáticas tienen como uno de sus objetivos la evaluación de los estudios científicos que pretenden ser incluidos en la revisión, se concluye que éstas cumplen con los criterios para poder ser consideradas como un proceso de selección artificial epistémica.

Palabras clave: revisiones sistemáticas, epistemología evolucionista, selección artificial del conocimiento, conocimiento científico.

\section{Abstract}

The aim of this essay is to conceptualize and analyze, from the framework of evolutionary epistemology, systematic reviews as a process of artificial selection of scientific knowledge in healthcare area. Firstly it is analyzed the main assumptions of the evolution of scientific knowledge by natural selection. Subsequently, it is proposed that systematic reviews be considered as a form of meta-research, the latter being understood as a type of research whose object of study is scientific knowledge and its evaluation. Afterwards, it is proposed the concept of Artificial Epistemic Selection to refer to a process of epistemic selection different from the one described by the model of natural selection. Since systematic reviews have as one of their objectives the evaluation of the scientific studies that intend to be included in the review, it is concluded that they satisfy the criteria to be considered as a process of artificial epistemic selection.

Keywords: systematic review, evolutionary epistemology, artificial selection of knowledge, scientific knowledge. 


\section{Introducción}

$\mathrm{L}$ as revisiones sistemáticas (RS) se han consolidado como un tipo de investigación fundamental para la identificación, evaluación, selección, descripción y síntesis de la evidencia obtenida a través de investigaciones primarias (empíricas) realizadas sobre un determinado objeto de estudio, en áreas como la medicina, la psicología clínica y le enfermería. Sin embargo, su estatus privilegiado dentro de la jerarquía de los diseños de investigación científica ha sido cuestionado por diversos autores (Bailar, 1997; Jansen, 2017; Greenhalgh, Thorne y Malterud, 2018), quienes han sometido a dura crítica el papel que las RS han jugado en el desarrollo del conocimiento científico.

Uno de los principales problemas a la hora de juzgar las contribuciones de las revisiones sistemáticas al proceso de evolución de la ciencia, es el de concebir a éstas como un mero medio para la identificación y agregación de la evidencia científica en un determinado campo de estudio (Edwards, 2014), sin considerar que los mecanismos de selección epistémica de las RS son esencialmente distintos a aquellos exhibidos por las investigaciones primarias, situación que influye sustancialmente sobre cómo se desarrolla el conocimiento, al igual que en las características de éste.

Así, mientras las investigaciones empíricas producen conocimiento a través de un proceso evolutivo de selección natural, es posible proponer que las RS siguen un proceso de selección artificial del conocimiento, toda vez que se observe que este tipo de revisiones propician un proceso de evolución dirigida, por medio de la identificación y selección de las mejores investigaciones científicas sobre un determinado problema, según criterios previamente establecidos (véase, por ejemplo, el uso indispensable de instrumentos para la evaluación de la calidad de investigaciones empíricas), esto con la finalidad de lograr la síntesis de la mejor evidencia disponible.

En este sentido, el presente ensayo tiene por objetivo conceptualizar y analizar, desde el marco de la epistemología evolucionista, a las revisiones sistemáticas como proceso de selección artificial del conocimiento científico en el área de la salud.

Para ello, se analizarán, en primera instancia, las características generales del conocimiento científico y sus diferencias con respecto a otras formas de conocimiento. Posteriormente, se hará una breve revisión de la epistemología evolucionista, como aproximación para entender el desarrollo del conocimiento científico. Asimismo, se expondrán las principales características de las revisiones sistemáticas y se discutirá el tipo de conocimiento obtenido a través de éstas, para, finalmente, discutir sus implicaciones en la evolución del conocimiento, como una forma de selección artificial epistémica, en el marco de la epistemología evolucionista. Para ilustrar la cuestión, se analizará un ejemplo particular de revisión sistemática en el área de la salud mental.

\section{Conocimiento científico y otras formas de conocimiento}

La ciencia ha sido presentada en diversas ocasiones, como la alternativa idónea para lograr conocimiento explicativo de la realidad. En este sentido, Bunge (2004) expresa que: 
... no se ha hallado nunca un método más poderoso que el de la ciencia, y todo esfuerzo en tal sentido que se haya visto coronado por el éxito ha resultado ser un perfeccionamiento de método científico; en particular, los intentos de captar la realidad directamente, sin elaboración alguna (o sea, por percepción directa, por simpatía o por pura especulación), han fracasado sin excepción. (pp. 27-28)

Debe entenderse, sin embargo, que el éxito explicativo, predictivo y descriptivo de la empresa científica no niega, de ninguna forma, los distintos tipos de conocimiento de los cuales hace uso el ser humano.

Para delimitar el conocimiento científico y distinguirlo de otras formas de conocimiento, se han propuesto distintos criterios de demarcación. Uno de los primeros intentos sistemáticos y rigurosos fue llevado a cabo por el positivismo lógico a principios del siglo XX, quienes encontraron en la verificación empírica de los enunciados el principal criterio de distinción entre la ciencia y la no ciencia. Sin embargo, para esta escuela filosófica, un enunciado tiene valor cognitivo si y sólo sí, puede verificarse empíricamente, negando, con ello, la posibilidad de cualquier otra forma de conocimiento, como el metafísico el cual es, en todo caso, un sistema de pseudoenunciados, que no representan más que un problema de mera confusión lingüística. En última instancia, todo enunciado teórico (es decir aquel tipo de enunciado que incluye conceptos teóricos no observables), debería ser, por lo tanto reductible a enunciados empíricos u observables, si se pretende que aquellos tengan el estatus de científicos.

El éxito de este programa filosófico no tardó en ser puesto en duda, ante la evidente incapacidad del mismo de dar una satisfactoria solución al problema de la demarcación científica. El problema de la inducción y el reduccionismo conceptual (Moulines, 2011), resaltaron ser dos de los principales obstáculos de dicho enfoque, volviéndose necesario idear alguna solución alternativa que pudiera sortearlos.

En este contexto, las críticas y aportaciones de Karl R. Popper (1962) resultaron fundamentales, sino para resolver definitivamente la cuestión, al menos para demostrar la existencia de posibilidades distintas a las planteadas por el positivismo a ultranza. Para Popper, el verificacionismo resultó ser un criterio de demarcación insatisfactorio, pues la inducción empírica de la cual partía, resultaba ser lógicamente insostenible, dado que nunca es posible verificar categóricamente un enunciado por medio de las observaciones de hechos particulares. Así, Popper considera a la falsación como la única alternativa lógicamente válida ante la verificación propuesta por los neopositivistas.

Por supuesto, la perspectiva popperiana no estuvo exenta de críticas, y tampoco fue la única alternativa a la visión neopositivista de la ciencia, sin embargo, no es la finalidad de este ensayo exponer a detalle cada una de ellas. El lector interesado puede encontrar exposiciones al respecto en Richards (2005), Cálves (2006) y Moulines (2011). Baste lo anterior para aclarar un punto importante: la ciencia y el conocimiento científico no son entidades que puedan describirse unidimensionalmente y es, por lo tanto, necesario observar distintas características para distinguirles de otras formas de conocimiento.

En el presente trabajo no se parte desde una perspectiva cientificista, sino que se reconocen diversas formas de conocimiento (por ejemplo, las artes), aun cuando sí se acepta la importancia fundamental de la ciencia cuando de explicar la realidad se trata.

En este orden de ideas, Cerón (2017) reconoce al menos cuatro formas distintas de conocimiento: 1) conocimiento acientífico; 2) conocimiento precientífico;3) conocimiento científico y, finalmente, 4) conocimiento meta-científico. El primero de estos tipos corresponde al conocimiento vulgar u ordinario, 
caracterizado por el sentido común y dirigido a la resolución de problemas cotidianos y prácticos. El segundo tipo, puede entenderse como un caso limítrofe entre el conocimiento acientífico y el científico propiamente dicho, sin embargo, el conocimiento precientífico puede no siempre desembocar en este último. Entonces, Cerón distingue dos formas de conocimiento precientífico, a saber: el protocientífico y el pseudocientífico. La primera de estas subdivisiones corresponde al conocimiento embrionario que, aunque cuenta con un objeto de estudio definido, puede no contar con el aparato teórico necesario para su estudio; por otro lado, el conocimiento pseudocientífico es todo aquel conocimiento que se presenta como científico cuando, en realidad, no lo es. La pseudociencia se caracteriza por la ausencia de rigor teórico y metodológico, así como de mecanismos de autocorrección característicos de la ciencia genuina.

En lo que respecta al tercer tipo de conocimiento, el científico, Cerón lo describe no recurriendo a un criterio único de demarcación, sino a un conjunto de características, a saber: es objetivo, metódico, sistemático y riguroso, con fines explicativos. No se trata, sin embargo, de una forma perfecta de conocimiento, sino más bien perfectible, susceptible de correcciones. De manera más específica, siguiendo a Ursua (1993), es posible identificar distintos criterios que caracterizan particularmente al conocimiento científico. En primera instancia, puede hablarse de criterios necesarios, es decir, que toda teoría con pretensiones científicas debe cumplir; estos, a su vez, se dividen en criterios lógicos (no circularidad, no contradicción interna ni externa), criterios de carácter científico (poder explicativo y testabilidad) y criterios de carácter semántico (éxito en los tests a los que las teorías son sometidas). Asimismo, contempla una serie de criterios deseables, que pueden servir para elegir entre teorías rivales que cumplan con todos los criterios necesarios. Estos son: generalidad, profundidad, precisión, simplicidad, intuitividad, capacidad predictiva y capacidad heurística.

Cerón (2017) distingue una última forma de conocimiento, que resultará ser de particular interés para la presente investigación, el llamado conocimiento metacientífico, el cual surge de la investigación que tiene por objeto de análisis y reflexión la realidad científica, indagando sobre los procesos de la ciencia, los factores contextuales que la determinan y sus productos. Encajan aquí diversas disciplinas como la filosofía de la ciencia y la sociología de la ciencia, entre otras. Se verá, además, que la revisión sistemática, concebida como un tipo de investigación, puede ser colocada, al menos parcialmente, en el contexto de este tipo de investigaciones y conocimiento, si bien no es una opinión común.

Conviene señalar que, si bien es posible encontrar otras formas de clasificar y caracterizar el conocimiento, la propuesta de Cerón resulta de particular valor puesto que toma como punto nodal al conocimiento científico, para, a partir de él, describir otras formas de conocimiento. Pero, además, al proponer una noción flexible de conocimiento metacientífico, es posible utilizar ésta para, como se ha sugerido, analizar las revisiones sistemáticas (una caracterización más restringida de la metaciencia puede encontrarse en Bunge (2004).

Por otra parte, el hecho de que la ciencia y el conocimiento científico sean utilizados como eje rector para la clasificación del conocimiento podría ser una decisión discutible, dado que, a primera vista, sugiere que el conocimiento científico es considerado como superior con respecto a otras formas de conocimiento. En el presente ensayo no se sostiene dicha posición, la cual, ciertamente, tampoco parece sostener Cerón (2017). Lo que si se acepta es que la ciencia nos provee una forma específica de entender el mundo y, posiblemente la más detallada y consistente hasta ahora (aunque no perfecta), si lo que se pretende es ahondar en los mecanismos explicativos de distintos objetos de estudio. En este sentido, se 
comparte el entusiasmo propio del realismo científico respecto a considerar a la ciencia como la mejor vía para entender la realidad, tanto en lo que se refiere a entidades observables como aquellas no observables $^{3}$. Dicho esto, es posible concebir otras formas de conocimiento como el conocimiento práctico, el filosófico, el artístico o el tecnológico, que distintos al científico, resultan ser relevantes para fines específicos, los cuales no necesitan ser los mismos que aquellos que persigue la ciencia.

Finalmente, para concluir esta breve caracterización del conocimiento en general y, particularmente, de la ciencia, es importante hacer una aclaración más. La ciencia misma puede clasificarse de distintas formas, según su objeto de estudio y métodos específicos. Una clasificación más o menos extendida, es la propuesta por Bunge (2004), quien distingue entre ciencias factuales y formales. La primera estudia ideas (por ejemplo: conjuntos, números, estructuras algebraicas, etcétera), mientras que la segunda estudia hechos (interacciones humanas, movimiento de electrones).

Mientras que en las ciencias formales se hace uso de un método enteramente deductivo partiendo de sistemas simbólicos "vacíos" (esto es, que son independientes de cualquier contenido suministrado por la experiencia), en las ciencias fácticas es necesaria la verificación de los postulados por medio de la observación de la realidad, pues dichos postulados refieren a hechos acaecidos en ésta (Bunge, 2001). Es importante resaltar que las ciencias fácticas o empíricas no se encuentran desligadas de las formales, pues aquellas se nutren de las herramientas lógicas y conceptuales que éstas últimas les proveen y, algunos problemas de carácter fáctico han propiciado desarrollos importantes en el terreno de lo formal.

Dicho lo anterior, conviene señalar que en el presente ensayo se pondrá énfasis solo en un tipo particular de ciencia, la ciencia fáctica o empírica, no porque las ciencias formales carezcan de interés epistémico, sino porque las revisiones sistemáticas de la literatura científica se han desarrollado y utilizado notoriamente para describir e integrar investigaciones de este tipo, antes que conceptuales (aun cuando, recientemente, el uso de revisiones sistemáticas se ha diversificado, llegándose a implementar incluso en campos como la computación, la bioética y la filosofía).

Habiendo pues, caracterizado, al menos sucintamente, a la ciencia y el conocimiento científico, es momento de analizar lo relativo al desarrollo de este último y los mecanismos implicados en el mismo. Para lograr esto, analicemos aquello que la epistemología puede decir al respecto.

\section{La epistemología evolucionista y el desarrollo del conocimiento científico}

El término epistemología ha tomado diversas acepciones a lo largo del tiempo. Algunos autores han optado por identificar a esta disciplina con la llamada filosofía de la ciencia (Bunge, 2002), atribuyéndole por objetivo el estudio de diversas problemáticas de índole lógica, ontológica, gnoseológica, metodológica y ética, en relación al quehacer científico.

La opinión del autor del presente ensayo, es la de considerar a la epistemología, en un sentido general, como el estudio del conocimiento; y, por lo tanto, de forma restringida, puede hablarse de una

3- Para una descripción detallada del realismo científico, véase Sankey (2008); reflexiones respecto al éxito explicativo de la ciencia y al progreso científico, desde esta misma postura, pueden encontrarse en Niiniluoto, (2017). 
epistemología de la ciencia, entendiendo a ésta como el estudio del conocimiento científico, y teniendo por objetivo comprender la naturaleza de este tipo de conocimiento, distinguirlo de otras formas de conocimiento, así como entender los medios por los cuales puede ser obtenido y justificado. Así pues, el presente trabajo se encuentra ubicado en el marco de una epistemología de la ciencia.

Una vez aclarada la terminología, resulta conveniente señalar también una distinción frecuente al momento de hablar de esta disciplina, a saber: la que existe entre la epistemología descriptiva y la epistemología normativa. La primera, se enfoca en resolver problemas relativos a la naturaleza del conocimiento, buscando dar respuestas a preguntas tales como ¿qué es el conocimiento?, ¿qué es la verdad?, ¿existe el conocimiento a priori? Etc.; por otra parte, la epistemología normativa, también llamada, metodología, busca comprender cuáles son las mejores maneras (si las hay) para obtener conocimiento, además de establecer criterios para distinguir qué puede considerarse como conocimiento válido (Bunge, 1983). Estas cuestiones suelen ser de suma relevancia al momento de estudiar el conocimiento en general, pero, además, son el interés particular de muchos de los estudios respecto a la ciencia.

Ahora bien, en la actualidad una de las principales problemáticas de interés epistemológico es aquella relativa a la evolución del conocimiento científico, pues, desde mediados del siglo XX surgió en los filósofos y científicos un interés por entender a la ciencia de forma contextualizada, observándola como una práctica humana y social producto de sus condiciones históricas y socioculturales. Por ello, diversos autores comenzaron a ocuparse de comprender los factores determinantes de la ciencia, así como el cambio conceptual y metodológico que ocurría en ésta. El llamado giro historicista iniciado con la publicación de la obra clásica La Estructura de las Revoluciones Científicas de Kuhn (1971), representó uno de los esfuerzos más importantes por remarcar la importancia que juega el estudio de la historia de la ciencia en el desarrollo, comprensión y contrastación de los modelos de cambio científico. Desde dicho enfoque, la ciencia, lejos de ser una entidad inmutable y perfecta, es caracterizada como un sistema cuya justificación no puede prescribirse, sino que ha de ser extraída de la observación de las distintas etapas históricas de la ciencia que ha llegado a considerarse exitosa (Cálves, 2006).

Por otra parte, una de las perspectivas más significativas en el estudio del conocimiento científico corresponde al llamado programa de epistemología naturalizada. Desde este enfoque se pretende que la ciencia, y otras formas de conocimiento, no han de ser analizadas desde la perspectiva apriorística y prescriptiva de la epistemología tradicional, sino que han de ser sometidas al escrutinio propio de los métodos de las ciencias naturales, pasando a ser así la epistemología un capítulo en alguna de dichas disciplinas (particularmente de las ciencias biológicas y cognitivas) (Rysiew, 2017).

En el seno de estas discusiones, se gesta la empresa intelectual conocida bajo el nombre de epistemología evolucionista (EE), concepto amplio (originalmente acuñado por Donald Campbell) que puede referirse a dos perspectivas interrelacionadas, pero distintas, respecto al conocimiento (Bradie y Harms, 2017):

1. El programa de la evolución de los mecanismos epistémicos (EEM, por sus siglas en inglés): una aproximación naturalista al estudio del conocimiento, desde la cual éste último se concibe como un producto de la evolución de los organismos (específicamente de sus funciones cognitivas), susceptible, por lo tanto, de ser estudiado por los métodos de las ciencias naturales. Debe observarse que, desde esta perspectiva, el conocimiento científico es sólo una de las diversas formas de conocimiento, siendo su objeto de estudio, pero no exclusivamente. 
2. El programa de la epistemología evolucionista de las teorías científicas (EET, por sus siglas en inglés): como una teoría evolutiva del conocimiento, particularmente científico, desde la cual se plantean una serie de analogías entre la evolución biológica de los organismos y la evolución de los sistemas conceptuales, tomándose prestados un conjunto de conceptos y planteamientos de la biología evolutiva. Desde esta perspectiva, el conocimiento científico toma un papel nodal, pasando a ser su principal objeto de estudio.

Si bien cada una de las perspectivas derivadas de las conceptualizaciones previas parte de presupuestos heterogéneos, existen puntos de convergencia importantes, pues como señala Wuketits (2001), todas ellas se relacionan por el énfasis que ponen sobre el problema del desarrollo de la mente y la consecución del conocimiento. En este orden de ideas, la evolución del conocimiento científico, puede entenderse como un episodio más en la larga cadena de evolución de los sistemas reales, que va desde la evolución cósmica, pasando por la evolución química, molecular y biológica, hasta llegar a los estadios más complejos como lo son la evolución cultural y la evolución del conocimiento científico (Ursua, 1993).

Sin embargo, puesto que el principal objetivo de este trabajo es dar cuenta de las implicaciones de las RS en la evolución del conocimiento, esta investigación se encontrará adscrita a la segunda de las perspectivas mencionadas (sin por ello negar la importancia de la primera). Por ello, será de principal importancia analizar los mecanismos a través de los cuales surgen las variaciones epistémicas (variaciones de conocimiento) y cómo estas dan pie al proceso evolutivo del conocimiento científico.

\subsection{E1 modelo evolucionista de selección natural del conocimiento científico}

Las principales premisas de la EET giran en torno a dos procesos fundamentales, a saber: la variación ciega y la retención selectiva. De acuerdo a Cziko (2001), dichos procesos son indispensables para todos los logros inductivos y para todo incremento genuino en el conocimiento, así como para el ajuste de un sistema a su entorno. El primero de estos términos, se ha caracterizado como el proceso, inintencionado, a través del cual se producen cambios en las teorías científicas. Pero tal apreciación se antoja incorrecta, toda vez que se analice la práctica científica como una actividad que implica agentes racionales (los investigadores científicos) que diseñan deliberadamente sus investigaciones. Por esta razón, King (2004) sugiere que una variación puede considerarse como ciega, no tanto por la carencia de intención en su inclusión a un sistema conceptual, sino, más bien "en el sentido de que su ocurrencia no está apoyada ni implicada por el conocimiento previo adquirido y aceptado en su área científica de adscripción" (p. 100). Esto es que, dado una determinada teoría (T), un problema (P) y una hipótesis $(\mathrm{H})$ como solución tentativa a $\mathrm{P}$, una variación se considere ciega con respecto a $\mathrm{P}$ si $\mathrm{y}$ sólo si (King, 2004):

1. T no implica que $H$ es una solución de $P$

2. T no es razón para la ocurrencia de $\mathrm{H}$ como solución de $\mathrm{P}$. 
Esto puede entenderse mejor al pensar en un científico que propone $n$ hipótesis alternativas como soluciones tentativas a un determinado problema. Si bien éstas se encuentran delimitadas o restringidas por la materia prima conceptual (T), de la cual provienen, su posibilidad de triunfo como solución al problema $\mathrm{P}$, no podrá ser deducida de antemano, sino hasta ser sometida a pruebas científicas rigurosas, siendo el proceso científico algo semejante a un proceso de tanteo por ensayo y error, a través de la postulación de múltiples alternativas sometidas a prueba. Aquellas alternativas que demuestren ser útiles para resolver efectivamente los problemas que se presentan, son retenidas por la comunidad científica (refiriéndose este hecho al segundo concepto mencionado, es decir, la retención selectiva) sin que esto impida la posibilidad de que vuelvan a ser sometidas a crítica y modificadas o sustituidas por nuevas alternativas más eficaces.

Dicho brevemente, el mecanismo de variación ciega-retención selectiva, actúa de la siguiente forma:

1. El investigador propone una solución alternativa (variación) a un determinado problema, sin tener seguridad del éxito de dicha solución (considérese que, de tener dicha certeza, no sería necesario realizar ninguna investigación para probar dicha variación)

2. De cumplir con su cometido, la variación es retenida, en caso contrario, es desechada.

\section{Metaciencia, metainvestigación y revisiones sistemáticas}

Para comprender el papel de las revisiones sistemáticas en la evolución del conocimiento científico es necesario diferenciarlas, en primera instancia, de la actividad científica. Anteriormente se hizo mención a un tipo de conocimiento conocido como metacientífico. La metaciencia, entendida de forma general como el estudio de la ciencia y sus productos, tiene distintas ramificaciones. Así, es posible catalogar dentro de este rubro a los intentos realizados desde la filosofía de la ciencia para comprender la naturaleza del conocimiento científico y sus métodos; la sociología de la ciencia, que estudia a la ciencia en tanto práctica social, cuyos productos y métodos se encuentran influenciados por aspectos de orden ideológico, cultural y/o políticos, entre otros.

Pero, además, dentro de la metaciencia es posible encontrar un tipo de disciplina conocida como metainvestigación, la cual es el estudio de la investigación en sí misma, incluyendo sus métodos, reportes, reproducibilidad, evaluación e incentivos, y cuyo objetivo es entender y mejorar la forma en cómo se realiza, comunica, verifica y evalúa la investigación científica, (Ioannidis, 2018). Ahora bien, las RS no suelen ser consideradas en sí mismas como formas de meta-investigación, aunque sí relacionadas (Ioannidis, 2018). La razón de esto es porque se suele considerar que éstas tienen por objetivo el conocimiento de un determinado objeto de estudio a través de las investigaciones primarias que se han hecho sobre el mismo. Desde tal concepción, el papel de las revisiones sistemáticas no parece estar vinculado con comprender la forma en cómo se realiza investigación y cómo mejorarla.

Pese a esto, en el presente trabajo se ha optado por considerar las RS como legítimas formas de metainvestigación, aun cuando su objetivo primordial sea obtener conocimiento científico sobre un determinado objeto de estudio. Para precisar esto, debemos observar que una revisión sistemática puede definirse como un tipo de investigación que tiene como fuente de datos la literatura científica existente 
sobre un determinado tema y cuyos objetivos son identificar, evaluar, describir y, en muchos de los casos, sintetizar, por medio de métodos estrictamente especificados e imparcialmente aplicados, los resultados de las investigaciones expuestas en la literatura en cuestión con la finalidad de dar respuesta a una determinada pregunta de investigación (Letelier et al., 2005; Sampaio y Mancini, 2007; Ferreira et al., 2011; Gough et al., 2012)

De la definición anterior podemos derivar los siguientes aspectos:

1. La revisión sistemática es un tipo de investigación documental. En efecto, no basta con considerarla como un tipo de técnica o método específico, sino como una legítima forma de investigación útil para alcanzar determinados objetivos y susceptible de ser diseñada de formas diversas, de acuerdo a las necesidades propias de cada problema que se pretenda resolver por medio de éstas ${ }^{4}$.

2. Si bien las RS son utilizadas para conocer un determinado objeto de estudio a través de la revisión de investigaciones primarias, la ejecución de una RS implica, necesariamente, la evaluación y reflexión sobre las características de las investigación que van a describirse o sintetizarse, esto con la finalidad de elegir las mejores investigaciones primarias en términos de calidad $^{5}$.

3. Considerando los aspectos 1 y 2, puede argumentarse que las RS son un tipo de investigación que tienen -al menos parcialmente- como objeto de estudio a la investigación científica en algunas de sus cualidades.

Así, en tanto que las RS tienen por objeto de análisis productos de investigaciones científicas, en términos de lo descrito en los puntos 1, 2 y 3 , resulta razonable identificarles como formas de metainvestigación, toda vez que aceptemos que esta última tiene por objeto la investigación científica. En este sentido, las RS proveen información útil para el mejor entendimiento de la investigación científica en un determinado campo, posibilitando también el identificar aspectos que mejorar. Cabe señalar que, si bien esta forma de entender las RS no es común, recientemente Murad, Asi, Alsawas y Alahdab (2016), han enfatizado el papel de estas en la evaluación de los estudios científicos, proponiendo concebirles no tanto como un tipo de investigación en la punta de la jerarquía de evidencia científica, sino más bien como un tipo de investigación cuya utilidad principal radica en poder evaluar la calidad de la evidencia.

Debe recalcarse que con esto no se pretende dejar de lado que el objetivo primordial de una revisión sistemática es proveer conocimiento científico sobre un objeto de estudio. Como se ha mencionado, una revisión sistemática pretende responder a determinada cuestión por medio de la síntesis de la evidencia científica respecto a una hipótesis, partiendo de supuestos de que la integración de los hallazgos de varios estudios empíricos individuales puede proveer una imagen más comprehensiva y concisa, que la que puede ofrecer un estudio empírico individual (Gough et al., 2012).

4- Para una exposición profunda sobre el carácter de las RS como investigación véase Gough et al. (2012).

5- Sobre la importancia de evaluar la calidad de los estudios incluidos en una RS así como los instrumentos utilizados para dicha tarea, véase, Cascaes et al., (2013); Zeng et al. (2015); y Carro (2017). 
Por lo tanto, en la presente investigación, se propone considerar a las revisiones sistemáticas como un tipo de investigación que tiene objetivos y características metacientíficas (metainvestigación), pero que, en mayor grado, busca generar conocimiento científico. Como puede observarse en el modelo propuesto en la figura 1, las RS tienen tanto un componente metacientífico como científico. Las columnas de color claro que se encuentran al fondo representan el componente metacientífico (M) y científico (C) de una revisión. La fase de descripción presenta tanto aspectos de $\mathrm{M}$ como de $\mathrm{C}$, representados por la columna más oscura, siempre que se observe que es posible realizar descripciones no sólo del conocimiento recolectado en las investigaciones científicas revisadas, sino también de las características de estas (métodos utilizados, fuentes de sesgo, técnicas de análisis, diseño, etcétera).

Gráfica 1

Proceso de una revisión sistemática

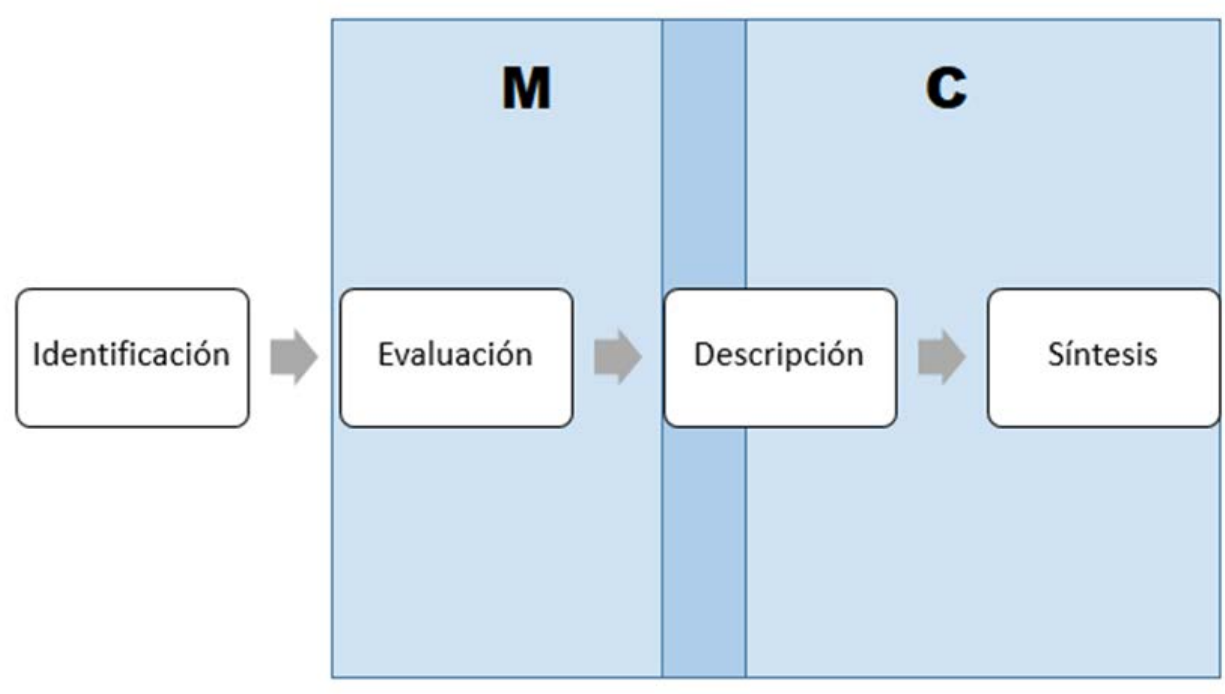

Fuente: elaboración propia.

\section{Las revisiones sistemáticas como un proceso de selección artificial del conocimiento científico}

Previamente se ha definido la evolución del conocimiento científico como un proceso de selección natural epistémica, vinculado a la ejecución de investigaciones empíricas. La noción de selección artificial, en el ámbito de la epistemología evolucionista, ha sido escasamente desarrollada. No obstante, es justo indicar que existen ciertos esfuerzos por aplicar el modelo selección artificial en el ámbito de la evolución del conocimiento, aunque un campo restringido como lo es la ciencia social aplicada, particularmente en lo que se refiere a la evaluación de programas de intervención social (Trochim, 2007; Brown et al., 2014). Sin embargo, con el auge de la metainvestigación y, particularmente de las RS, parece pertinente tomar en cuenta esta noción no sólo para el análisis de un campo tan específico 
como al anterior, sino también para abordar el problema de la evaluación y desarrollo del conocimiento científico en general, ya que, como se verá a continuación, una noción como esa permite dar cuenta de un proceso de evolución del conocimiento esencialmente distinto al descrito por el modelo de selección natural.

En primera instancia conviene considerar aquellos aspectos que distinguen a la selección artificial. En biología, dicha forma de selección, es un tipo de crianza controlada y llevada a cabo por seres humanos, la cual permite alterar las características genéticas de poblaciones domésticas, basándose en el valor percibido que tienen dichas características heredadas (Mai et al., 2005) y generalmente teniendo por objetivo la mejora de dichas poblaciones. La selección artificial se distingue de la selección natural, por el hecho de que en la primera interviene un componente de deliberación en la selección de las características heredables, siendo estas plenamente conocidas, con miras a provocar un proceso de evolución dirigido.

Recuérdese que la evolución del conocimiento científico, por selección natural, se da a través de un mecanismo de variación ciega-retención selectiva, por medio del cual se produce un cambio en algún sistema conceptual, en el cual el investigador no tiene certeza alguna de que las variaciones introducidas en los sistemas conceptuales tendrán éxito en la explicación de un determinado problema. Sin embargo, como se ha mencionado, el desarrollo cada vez mayor de esfuerzos provenientes de la metainvestigación, vuelve necesario repensar la forma en cómo actualmente el conocimiento científico evoluciona.

Por ello, con base en la distinción entre selección natural y artificial, se entenderá por selección artificial del conocimiento científico, o selección artificial epistémica (SAE), aquel proceso por el cual los investigadores deliberadamente identifican y seleccionan las mejores investigaciones, de acuerdo a criterios de evaluación determinados (tipo de investigación, calidad metodológica, etcétera), con la finalidad de reproducirlas y/o sintetizarlas, para obtener un mejor conocimiento sobre una determinada cuestión.

La noción anterior no contraviene, de forma alguna, el concepto de selección natural del conocimiento científico, el cual se desarrollará en la medida que se siga llevando a cabo investigación empírica. En este orden de ideas, es importante observar que, tanto la selección natural como la selección artificial, se fundamentan en el mecanismo de variación ciega-retención selectiva del conocimiento, pero la selección artificial incorpora, además el componente de evaluación deliberada de piezas de investigación en las cuales se van presentando las variaciones.

Por otra parte, el término artificial que acompaña a esta forma de selección epistémica no debe ser interpretado en un sentido negativo, pues es utilizado únicamente para referir a un proceso de selección distinto al de selección natural, ambos entendidos en el marco de la epistemología evolucionista adoptado en este ensayo.

Así, lo que la noción de SAE nos permite resaltar es que, actualmente, resulta limitado concebir la evolución del conocimiento científico únicamente en términos de selección natural, debido a que esta evolución también puede darse en función de la evaluación concienzuda de las características de las investigaciones y con miras a obtener la mejor forma de conocimiento posible. Ambos tipos de selección operan, por supuesto, sobre características epistémicas, y permiten obtener conocimiento científico. Pero a pesar de las similitudes, ambos procesos son distintos. 


\subsection{Un caso para ilustrar la SAE}

Las distintas investigaciones que se realizan ante una determinada problemática, por ejemplo la efectividad de un tratamiento ante una determinada enfermedad, suelen ser réplicas que buscan corroborar los resultados de la investigación que confirmó la variación triunfante. Sin embargo, éstas réplicas pueden y, de hecho incluyen, cambios en la población de estudio, los instrumentos utilizados para medir variables, los diseños de investigación e inclusive el tipo de análisis de datos realizados, siendo todos estos aspectos que influyen en la validez de las conclusiones de una investigación.

Al realizar una revisión sistemática, se consideran criterios de calidad y elegibilidad que las investigaciones que pretenden incluirse en la revisión deben cumplir. A partir de estos criterios se determina cuáles investigaciones son valiosas para ser incluidas y cuáles son aquellas de las que se puede prescindir. Si es el caso, los resultados incluidos en las investigaciones son sintetizados mediante un método específico (v.g. metaanálisis). De esta forma, solamente serán caracterizados y sintetizados aquellos resultados provenientes de investigaciones que cumplan con los criterios de elegibilidad y calidad establecidos previamente por el investigador. Las decisiones en materia de investigación científica y práctica, serán guiadas en gran medida por estos criterios. Por lo tanto, el considerar como pertinentes únicamente estudios aleatorizados (experimentos), conlleva dejar de lado otras especies de investigación que no se apegan a dicho criterio. Esto, por supuesto, trae como consecuencia la perpetuación de ciertas formas de conocimiento, las cuales terminan por ser el resultado de un proceso de selección deliberado.

Un ejemplo real puede ayudar a ilustrar mejor esta cuestión. Recientemente, Méndez-Bustos et al. (2019), llevaron a cabo una revisión sistemática para determinar la efectividad de ciertas formas de psicoterapia en el tratamiento de pacientes con riesgo de cometer suicidio. Para el caso particular de dicha revisión, los autores incluyeron únicamente estudios observacionales en los cuales se utilizara algún enfoque terapéutico para ayudar a poblaciones que presentaran ideación suicida, planes suicidas o algún intento de suicidio. En este sentido, estudios aleatorizados fueron excluidos completamente del estudio. Para evaluar la calidad de los estudios observacionales los autores utilizaron la Quality Assessment Tool for Observational Cohort and Cross-Sectional Studies. Los autores concluyen que la terapia cognitivo conductual y la terapia dialéctica conductual son las formas más eficaces de psicoterapia ante pacientes con riesgo suicida.

Es importante observar que esta conclusión, entendida como un aporte al conocimiento sobre el tema en cuestión, ha dependido en gran medida de aquellos criterios y decisiones tomadas por los investigadores que llevaron a cabo la revisión. La exclusión de estudios experimentales, el uso de ciertos criterios de calidad y el interés por variables específicas de las poblaciones analizadas, son solo algunos de los aspectos que tienen un impacto importante sobre las conclusiones obtenidas, las cuales se asumen, sin embargo, que son lo suficientemente adecuadas en la medida que se extrajeron de la revisión de investigaciones que lograron cumplir con criterios de elegibilidad y calidad.

De manera análoga a lo que ocurre en el caso de la selección artificial de organismos, lo que se pretende es que el conocimiento obtenido a través de una revisión sistemática sea lo mejor posible, siendo la principal guía para lograr esto, los criterios evaluativos predefinidos por los investigadores. Si una nueva variación en el conocimiento es aceptada a través del cumplimiento de estos, puede considerarse que ha evolucionado por selección artificial. 


\section{Conclusión}

En el presente ensayo se han delineado dos propuestas fundamentales: 1) la concepción de la revisión sistemática como un tipo de metainvestigación y 2) la concepción de la revisión sistemática como un proceso de selección artificial del conocimiento científico. Esta forma de selección se ha definido como un proceso por el cual los investigadores deliberadamente identifican y seleccionan las mejores investigaciones, de acuerdo a criterios de evaluación determinados (tipo de investigación, calidad metodológica, etcétera), con la finalidad de reproducirlas y/o sintetizarlas, para obtener un mejor conocimiento sobre una determinada cuestión.

Se ha señalado que ambas propuestas se encuentran relacionadas, siendo imposible concebir la segunda sin la primera. Como se ha mencionado, el componente metacientífico de la revisión sistemática se detecta en aquellas actividades de evaluación crítica que componen a toda revisión de este tipo, y que son necesarias para describir e integrar la mejor evidencia posible.

La evaluación de las investigaciones que pretenden ser incluidas en una revisión (la cual, claro está, tiene por objetivo aportarnos conocimiento sobre una determinada cuestión), constituye el fundamento de lo que en el presente ensayo se ha detectado como un proceso de selección artificial del conocimiento científico. Puesto que dicha evaluación parte de criterios previamente establecidos por el investigador, el conocimiento obtenido por medio de una revisión sistemática está determinado por estos criterios los cuales, se asume, que de ser cumplidos darán como resultado una mejor forma de conocimiento. Así, la valía de éste no queda determinada por la puesta a prueba empírica que corresponde al proceso de selección natural, sino por los criterios que el revisor o revisores considera como idóneos o pertinentes para ser cumplidos por las investigaciones.

Esta situación ha tenido bastantes implicaciones en la síntesis de la evidencia científica y la Práctica Basada en Evidencia, particularmente en el ámbito de la salud. Por ejemplo, en diversas ocasiones se ha criticado la tendencia a incluir únicamente ensayos clínicos aleatorizados e investigaciones cuantitativas en las revisiones sistemáticas, razón por la que cada vez es más frecuente exhortar a la inclusión de investigaciones de corte cualitativo, y metodologías no experimentales.

Claro está que la noción de selección artificial previamente descrita corresponde a un mero esbozo especulativo. Es importante notar que una limitación para la consolidación de dicha noción es el escaso interés que, desde la epistemología evolucionista, se le ha dado a la evolución del conocimiento por selección artificial, lo que dificulta el partir de un marco teórico sólido. Por otro lado, si bien la presente propuesta se ha enfocado en caracterizar a las revisiones sistemáticas como un proceso de selección artificial epistémica, no quiere decir que sea la única forma de llevar a cabo un proceso como este. Los esfuerzos de Trochim (2007) y Brown et al., (2014), constituyen buenos ejemplos de esto.

Entonces, la principal contribución de este trabajo es, al menos, plantear la posibilidad de concebir la evolución del conocimiento científico de una forma más cercana a la actual práctica científica, así como coadyuvar a la conceptualización del papel que juegan las revisiones sistemáticas en la generación del conocimiento como formas genuinas de investigación. 


\section{Referencias}

Bailar, J. (1997). The promise and problems of meta-analysis. New England Journal Medicine, 337, 559561. https://doi.org/10.1056/NEJM199708213370810

Bunge, M. (1983). Treatise on Basic Philosophy (vol. 5). D. Reidel Publishing Company.

Bunge, M. (2001). La ciencia, su método y su filosofía. Sudamericana.

Bunge, M. (2002). Epistemología (4ª ed.). Siglo XXI Editores.

Bunge, M. (2004). La investigación científica, su estrategia y su filosofía (3a. ed.). Siglo XXI Editores.

Bradie, M. y Harms, W. (2017). Evolutionary Epistemology. En E. N. Zalta (Ed.), The Stanford Encyclopedia of Philosophy (Spring 2017 ed.). https://plato.stanford.edu/entries/epistemology-evolutionary

Brown, J., Hargraves, M. y Trochim, W. M. (2014). Evolutionary Evaluation: Implications for evaluators, researchers, practitioners, funders and the evidence-based program mandate. Evaluation and Program Planning. 45, 127-139. https://doi.org/10.1016/j.evalprogplan.2014.03.011

Cálves, J. (2006). Modelos de cambio científico a partir de la selección natural: análisis y propuestas. Llull Revista de la Sociedad Española de Historia de las Ciencias y las Técnicas, 29, 221-257.

Carro, E. H. (2017). La calidad del reporte científico. Apuntes sobre la importancia, protocolos y recomendaciones. Revista de Psicología y Ciencias del Comportamiento de la Unidad Académica de Ciencias Jurídicas y Sociales, 8(2). 1-5. http://dx.doi.org/10.29365/rpcc.20171229-57

Cascaes, F., Valdivia, B. A., da Rosa, R., Babosa, P. J. y da Silva, R. (2013). Escalas y listas de evaluación de la calidad de estudios científicos. Revista Cubana de Información en Ciencias de la Salud, 24(3), 295-312. http://dx.doi.org/10.36512/rcics.v24i3.438

Cerón, A. (2017). Cuatro niveles de conocimiento en relación a la ciencia. Una propuesta taxonómica. CIENCIA Ergo-Sum, 24(1), 83-90. https://doi.org/10.30878/ces.v24n1a9

Cziko, G. (2001). Universal Selection Theory and The Complementarity of Different Types of Blind Variation and Selective Retention. En C. Heyes y D. Hull (Eds.) Selection Theory and Social Construction: The Evolutionary Naturalistic Epistemology of Donald T. Campbell (pp. 15-35). SUNY Press.

Edwards, B. (2014). What is the value of a systematic review? Journal of Shoulder and Elbow Surgery, 23(1), 1-2. https://doi.org/10.1016/j.jse.2013.09.001

Ferreira, I., Urrútia, G. y Alonso-Coello, P. (2011). Systematic reviews and meta-analysis: scientifc rationale and interpretation. Revista Española de Cardiología, 64(8), 688-696. https://doi.org/10.1016/j. recesp.2011.03.029

Greenhalgh, T, Thorne, S y Malterud, K (2018). Time to challenge the spurious hierarchy of systematic over narrative reviews? European Journal of Clinical Investigation, 48(6), e12931. https://doi. org/10.1111/eci.12931

Gough, D., Oliver, S. y Thomas, J. (2012). Introducing systematic reviews. En D. Gough, S. Oliver y J. Thomas (Comp.). An introduction to Systematic Reviews (pp. 1-16). Sage.

Ioannidis, J. (2018). Meta-research: Why research on research matters. PLOS Biology, 16(3), e2005468. https://doi.org/10.1371/journal.pbio.2005468

Jansen, S. (2017, Fall). Bias within systematic and nonsystematic literature reviews: the case of the Balanced Scorecard [Master's thesis, University of Twente]. Recuperado de: https://essay.utwente.nl/73771/1/ Jansen_MA_BMS.pdf 
King, P (2004). La noción de "variación ciega" en el ámbito del cambio científico: una defensa. Diánoia, 49(53), 93-110. https://doi.org/10.21898/dia.v49i53.399

Kuhn, T. (1971). La estructura de las revoluciones científicas. Fondo de Cultura Económica.

Letelier, L. M., Manriquez, J. J. y Rada. G. (2005). Revisiones sistemáticas y metaanálisis: ¿son la mejor evidencia? Revista Médica Chilena, 133, 246-249. http://dx.doi.org/10.4067/S003498872005000200015

Mai, L. L., Young, M. y Kersting, P. M. (2005). The Cambridge Dictionary of Human Biology and Evolution. Cambridge University Press.

Méndez-Bustos, P., Calati, R., Rubio-Ramirez, F., Olié, E., Courtet, P. y Lopez-Castroman, J. (2019) Effectiveness of Psychotherapy on Suicidal Risk: A Systematic Review of Observational Studies. Frontiers in Psychology, 10. https://doi.org/10.3389/fpsyg.2019.00277

Moulines, U. (2011). El desarrollo moderno de la filosofía de la ciencia (1890-2000) (1era ed.). Universidad Nacional Autónoma de México; Instituto de Investigaciones Filosóficas.

Murad, M. H., Asi, N., Alsawas, M. y Alahdab, F. (2016). New evidence pyramid. Evidence-Based Medicine, 21(4), 125-127. https://doi.org/10.1136/ebmed-2016-110401

Niiniluoto, I. (2017). Scientific progress. En J. Saatsi (Ed.). The Routdlege Handbook of Scientific Realism. (pp. 187-199). Routledge.

Popper, K. (1962). La lógica de la investigación científica. Tecnos.

Richards, S. (2005). Filosofía y sociología de la ciencia (5ta ed.). Siglo XXI Editores.

Rysiew, P. (2017). Naturalism in Epistemology. En E. N. Zalta (Ed.), The Stanford Encyclopedia of Philosophy (Spring 2017). https://plato.stanford.edu/archives/spr2017 /entries/epistemology-naturalized

Sampaio, R. F. y Mancini, M. C. (2007). Systematic review studies: a guide for careful synthesis of scientifc evidence. Revista Brasileña de Fisioterapia, 11(1), 77-82. https://doi.org/10.1590/S141335552007000100013

Sankey, H. (2008). Scientific Realism and the Rationality of Science. Ashgate.

Trochim, W. M. (2007). Evolutionary Perspectives in Evaluation: Theoretical and Practical Implications. 30th Annual Conference of the Eastern Evaluation Research Society, Absecon. https://www.socialresearchmethods.net/research/EERS2007/Evolutionary\%20Perspectives\%20in\%20Evaluation\%20 Theoretical\%20and\%20Practical\%20Implications.pdf

Ursua, N. (1993). Cerebro y conocimiento: un enfoque evolucionista. Anthropos.

Wuketits, F. (2001). The philosophy of Donald T. Campbell: A short review and critical appraisal. Biology and Philosophy, 16(2), 171-188. https://doi.org/10.1023/A:1006721104642

Zeng, X., Zhang, Y., Kwong, J. S., Zhang, C., Li, S., Sun, F., Niu, Y. y Du, L. (2015). The methodological quality assessment tools for preclinical and clinical studies, systematic review and meta-analysis, and clinical practice guideline: a systematic review. Journal of Evidence-Based Medicine, 8(1), 2-10. https://doi.org/10.1111/jebm.12141 

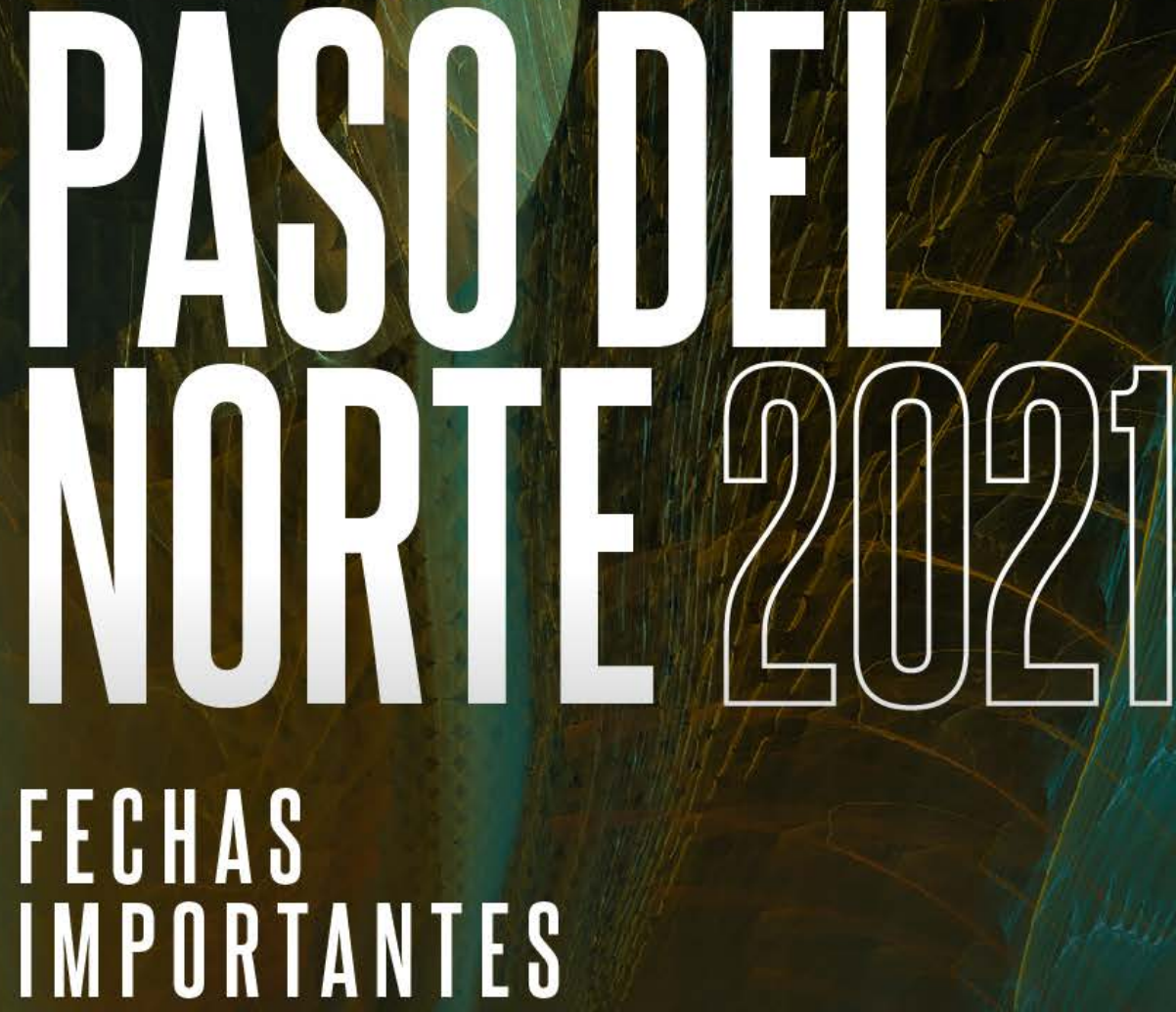

Recepción de trabajo

Del 15 de febrero al 7 de junio

Publicación de propuestas aceptadas 30 de julio

\begin{tabular}{ll}
\hline Fecha límite envío ponencias in extenso & $\mathbf{3 0}$ de septiembre \\
\hline Fecha límite de inscripción para ponentes & $\mathbf{8}$ de octubre \\
\hline Publicación del programa del Congreso & $\mathbf{2 2}$ de octubre \\
\hline Realización del Congreso & del $\mathbf{8}$ al $\mathbf{1 1}$ de noviembre \\
\hline Fecha límite para trámite de constancias & $\mathbf{3 0}$ de noviembre
\end{tabular}

Fecha límite para trámite de constancias

30 de noviembre

Registro de trabajos:

http://info.uacj.mx/congresocs2021 